\title{
Network Control and Management Challenges in Opaque Networks Utilizing Transparent Optical Switches
}

\author{
G. Ellinas ${ }^{\llbracket, ~ J-F . ~ L a b o u r d e t t e *, ~ J . ~ W a l k e r ~}{ }^{\#}$, S. Chaudhuri*, L. Lin ${ }^{\dagger}$, E. Goldstein ${ }^{\dagger}$, K. Bala* \\ "Department of Electrical Engineering, City College, New York, NY 10031 \\ *Tellium Inc., 2 Crescent Place, Oceanport NJ 07757 \\ \#JayWalker Technical Consulting, Oceanport, NJ 07757. \\ ${ }^{\dagger}$ Department of Electrical Engineering, University of Washington, Seattle, WA 98195
}

\begin{abstract}
There is a potential for significant cost, footprint and power savings by eliminating unnecessary OptoElectronic (OE) conversions on a signal path in a core optical mesh network. This paper addresses and clarifies some fundamental issues surrounding all-optical networking and all-optical switching and analyzes the tradeoffs between transparent (no OE conversions) and opaque networking (with OE conversions). It investigates a number of networking and interface compatibility issues that arise for an opaque network with transparent switches and presents a number of ways on how this issues can be addressed.
\end{abstract}

\section{Introduction}

To carry out our assessment of opaque and transparent networks, we make the following basic assumptions on the requirements for core mesh networks:

- Network operators require lowest cost network not just lowest cost network element. A network without wavelength conversion in the optical domain and without tunable wavelength access could lead to higher network cost due to inefficient capacity usage even though the network elements may be cheaper than their counterparts with wavelength conversion in the electrical domain.

- A network operator must not be constrained to buy the entire network from a single vendor.

- In order to build a dynamic, scalable and manageable backbone network it is essential that manual configuration be eliminated as much as possible. This requires automatic port/neighbor and network topology discovery and other networking functions such as service assurance (e.g. access point performance monitoring for Service Level Agreement (SLA) verification), interworking with other network equipment (e.g., unequipped signal), fault management, and performance management regardless of the switching technology. 
- An optical switching system must be easily scalable with low cost and small footprint as the network grows to many hundreds of wavelength channels per fiber and to a speed of $40 \mathrm{~GB} / \mathrm{s}$.

Based on these requirements we identify the challenges faced by completely transparent core mesh networks. The results of this exercise tend to indicate that core mesh networks will remain opaque for some time. This paper then explores the potential opportunity for cost reduction and scalability by introducing transparent switches in opaque networks. However, several challenges, both in technology and in network architecture, will have to be addressed before one can achieve the potential benefits of transparent switches.

The paper is organized as follows: Section 2 describes the possible choices for network architectures, Section 3 discusses the challenges associated with a completely transparent network architecture and Section 4 addresses the advantages and drawbacks of an opaque network architecture. Section 5 investigates the control and management issues that arise in opaque network architectures with opaque or transparent switches and Section 6 briefly discusses switch fabrics for transparent switches. Finally, Section 7 addresses the role of transparency in the near future and Section 8 offers some concluding remarks.

\section{Network Architectures}

Increased traffic volume due to the introduction of new broadband services is driving carriers to the deployment of an optical transport layer based on Wavelength Division Multiplexing (WDM) [1]. The network infrastructure of existing core networks is currently undergoing a transformation from rings using SONET Add/Drop Multiplexers (ADMs) to mesh topologies using Optical Cross-connects (OXCs). A core optical network architecture can be opaque or transparent. An opaque architecture means that the optical signal carrying traffic undergoes an Optical to Electronic to Optical (OEO) conversion at different places in the network. A transparent architecture means that the optical signal carrying traffic stays in the optical domain from the time it is generated at the edge of the network until it leaves the network. 
Even though the applications driving the large scale deployment of transparent optical switches are not currently in place (niche applications in today's networks only use a very small number of transparent switches), and the traffic demand does not currently justify the use of transparent switches that are cost effective at very high bit rates, it is possible that at some point in the future transparent switches may be deployed in the network. Based on this assumption, this paper explores the network control and management issues that arise when transparent switches are introduced in opaque networks. 3D MEMS-based switch fabrics are the ones that have offered the most viable approach to make single-stage switch fabrics with large port counts even though they are not quite ready for the deployment of transparent switches in the network.

Figure 1 illustrates the four different node architectures that can comprise a reconfigurable core optical network. The first architecture shows a fixed patch panel. Fixed patch panels located between WDM systems with transponders are currently being replaced by opaque (OEO) switching nodes (with electrical switch fabrics), as shown in architecture of Figure 1(b), due to their complete lack of flexibility. This is an opaque network architecture, as the optical signal undergoes OEO conversions, with an opaque OEO switch [2]. The third architecture shows a transparent (OOO) switch between WDM systems with transponders that is complemented by an OEO switch for drop traffic. This is again an opaque network architecture, as the optical signal undergoes OEO conversions at the WDM transponders. The fourth architecture shows a completely transparent network topology, consisting of transparent optical switches and WDM systems that contain no transponders. The transparent switch would be complemented as in 1(c) by an OEO switch for drop traffic. In this architecture, the signal stays in the optical domain until it exits the network. 


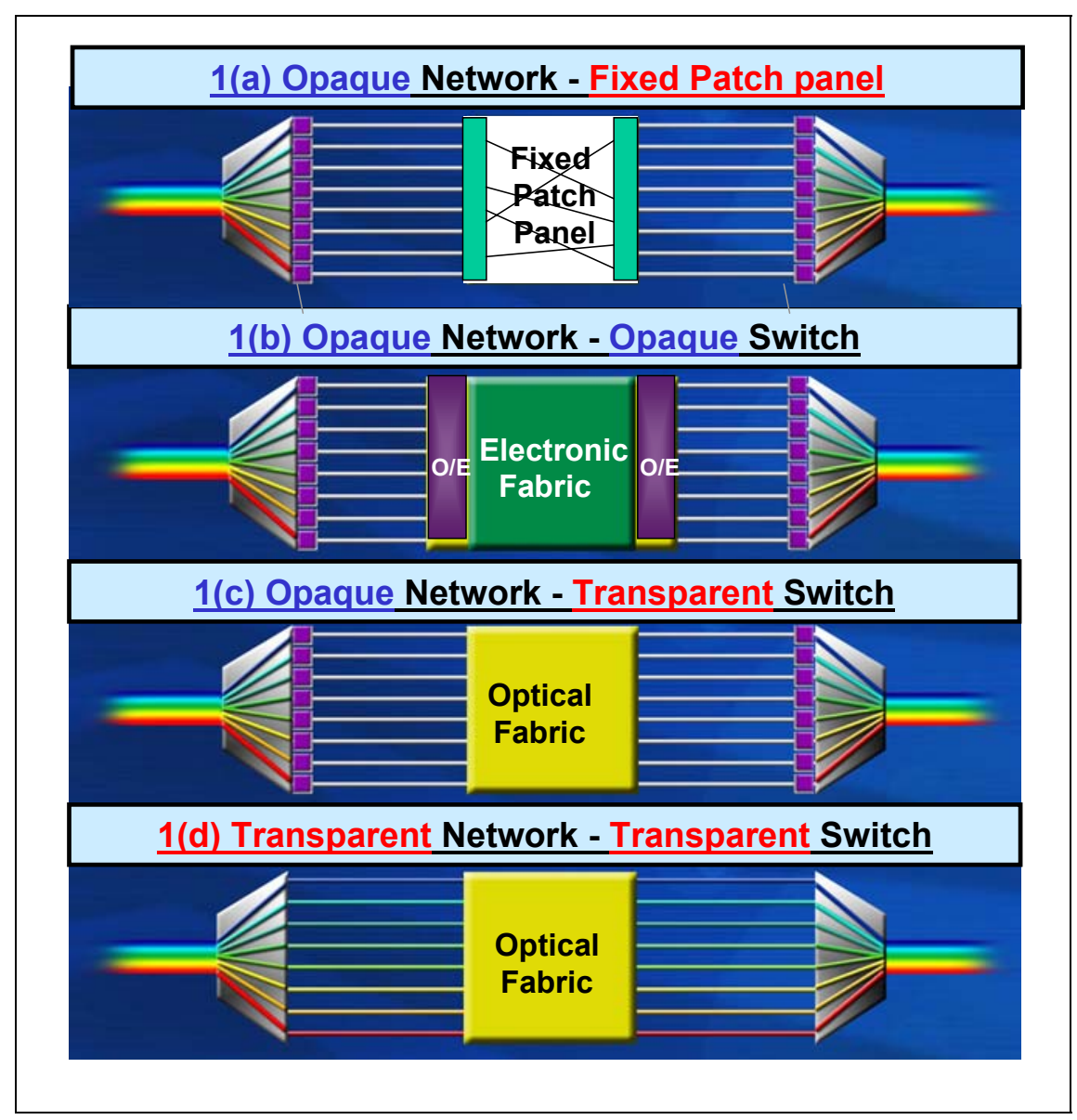

Figure 1: Node Architectures for a Core Optical Network

\section{Transparent Network Architecture}

The transparent network shown in Figure 1(d) and elaborated on in Figure 2 is a seemingly attractive vision. A signal (wavelength) passing through an office does not undergo optoelectronic conversion. Similarly, a client Network Element (NE), such as a router, interfaces with the switch using long-haul optics to interface with the WDM equipment without any $\mathrm{O} / \mathrm{E}$ conversion. Since a signal from a client NE connected via a specific wavelength must remain on the same wavelength when there is no wavelength conversion, only a small size switch fabric is needed to interconnect the WDMs and NEs in a node. This architecture also implies end-to-end bit rate and data format transparency. Note that another architecture of a transparent switch in a transparent network may include a single large fabric instead of multiple switch matrices of small port counts. If one is to provide flexibility, such an architecture design would require the use of tunable lasers at the clients and wavelength conversion. 


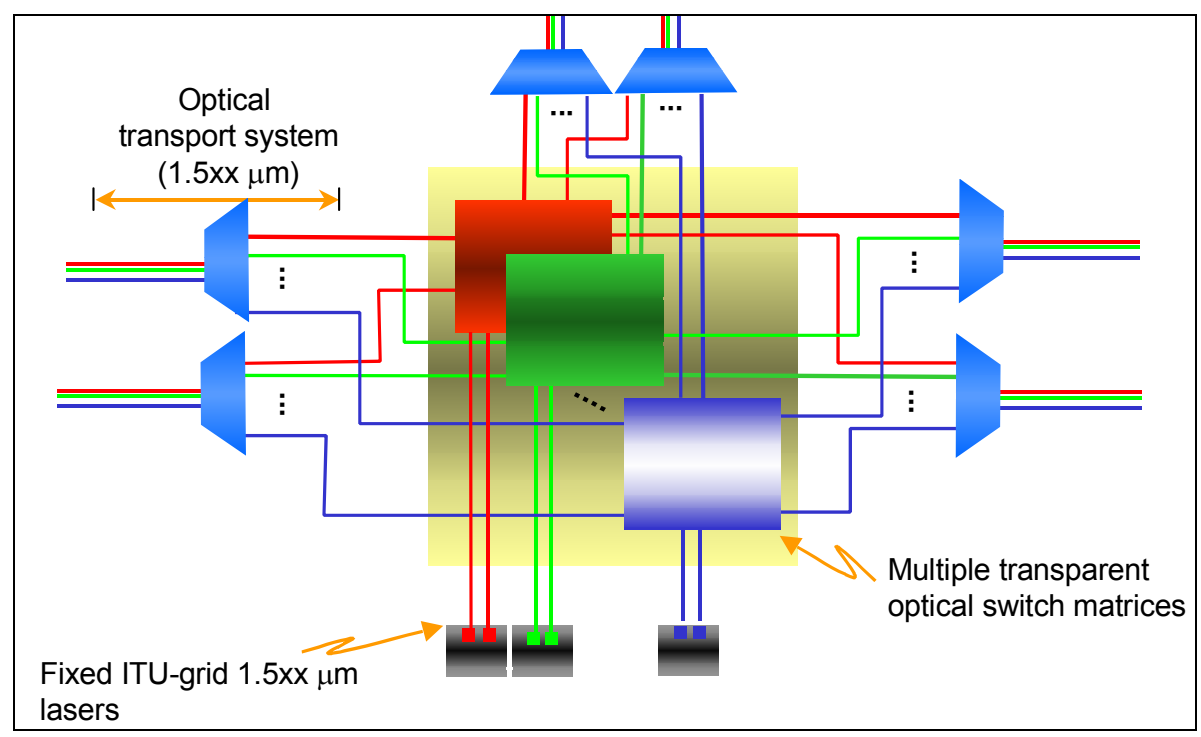

Figure 2: Transparent switch architecture in a transparent network

This network may provide significant footprint and power savings and on the surface suggests cost savings. However, while the transparent network architecture may be a viable option for small-scale networks with pre-determined routes and limited numbers of nodes, it is not a practical solution for a core mesh network for the following reasons:

1. This network does not allow wavelength conversion ${ }^{1}$ thus essentially creating a network of $n$ ( $n$ being the number of WDM channels) disjoint layers. Inflexible usage of wavelengths in this network would lead to increased bandwidth and network operational cost, thus negating all savings that may result from elimination of optoelectronic conversion. In addition, for this technology to be effective and in order to build a flexible network for unrestricted routing and restoration capacity sharing, an all-optical 3R-regeneration ${ }^{2}$ function must be available. Currently such a technology that can be harnessed in a commercial product does not exist [3].

2. In the absence of wavelength conversion, only client-based $1+1$ dedicated protection can be easily provided $[4,5]$. The wavelength continuity constraint on backup paths makes resource sharing almost impossible in transparent networks and consequently no shared mesh restoration can be easily offered. This in turn means that the capacity

\footnotetext{
${ }^{1}$ Our assumption here is that there will be no commercially viable wavelength conversion technology in the optical domain available in the next several years.

${ }^{2} 3 \mathrm{R}$ function implies retiming, reshaping and regeneration of the signal.
} 
requirement for protected services is significantly higher (80-100\%) for transparent compared to opaque networks [6].

3. Physical impairments such as chromatic dispersion, polarization mode dispersion (PMD), fiber non-linearities, polarization dependent degradations, WDM filter passband narrowing, component crosstalk, amplifier noise, etc. accumulate over the physical path of the signal due to the absence of opto-electronic conversion. The accumulation of these impairments requires engineering of end-to-end systems in fixed configurations [7-10]. It is thus not possible to build a large network with an acceptable degree of flexibility.

4. The design of high-capacity DWDM systems is based on intricate proprietary techniques, eluding any hope of interoperability among multiple vendors in the foreseeable future. Since the interface optics at the client NE launches a signal through the all-optical switch directly into the WDM system without O/E conversion, and it is not possible to develop a standard for the interface for a high capacity WDM, the operators will not have the flexibility to select the client NE vendor and the WDM vendor independently. Consequently, transparent networks by necessity are single vendor (including the client network elements) solutions.

5. Finally, in addition to all the limitations discussed above, the challenge of performance-engineering continental-scale transparent reconfigurable wavelengthrouted networks remains severe and, in networks that push limits, remains unsolved despite some attempts at formalizing the routing problem [11].

It is apparent that a number of key carrier requirements - dynamic configuration, wavelength conversion, multi-vendor interoperability of transport equipment (WDM), low network-level cost - would be very hard to meet in a transparent network architecture. Therefore, an opaque network solution will remain for now the only practical and cost-effective way of building a dynamic, scalable, and manageable core backbone network. 


\section{Opaque Network Architecture}

Even though the opaque network solution may be more expensive in terms of equipment costs when the core network capacity increases significantly, the opaque network offers the following key ingredients for a large-scale manageable network:

1. No cascading of physical impairments. This eliminates the need to engineer end-toend systems (only span engineering is required) and allows full flexibility in signal routing.

2. Multi-vendor interoperability using standard intra-office interfaces (see Figure 3).

3. Wavelength conversion enabled. Network capacity can be utilized for service without any restrictions and additional significant cost savings can be offered by sharing restoration capacity in a mesh architecture (see Figure 3).

4. Use of an all-optical switch enabled without any compromise of the control and management functions. Overhead visibility (available through the OEO function that complements the OOO switch) provides support for the management and control functions that are taken for granted in today's networks.

5. The network size and the length of the lightpaths can be large, since regeneration and re-timing is present along the physical path of the signal.

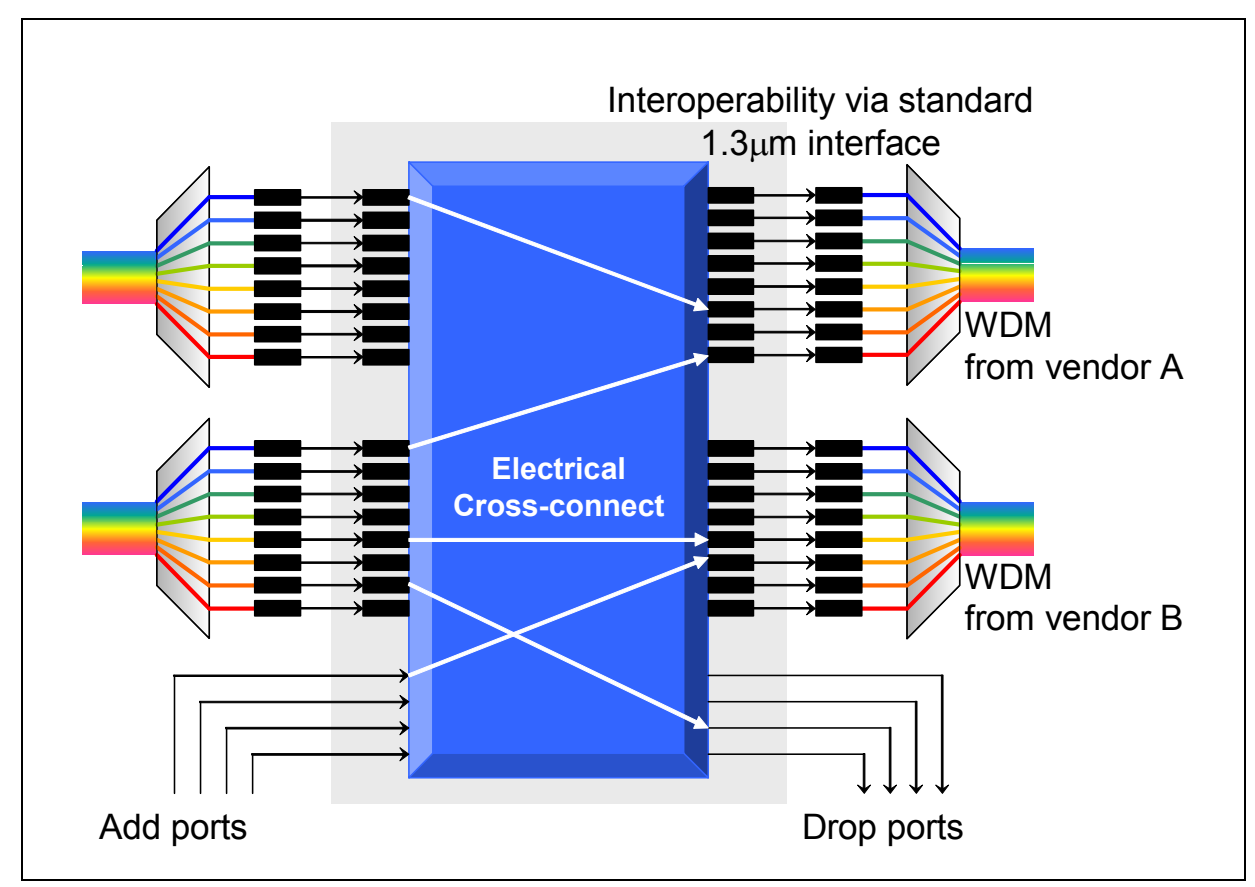




\section{Figure 3: Multi-vendor interoperability and wavelength translation as a by-product of an opaque network architecture}

Having shown that transparent core mesh network architectures are likely to remain unrealistic for quite some time, we now turn our attention to opaque network architectures in which WDM systems contain transponders. Today's architecture contains opaque switches (with an electronic switch fabric) in an opaque network (with transponders present in the WDM system). This architecture is shown in Figure 4. The interfaces to the fabric are opaque interfaces, which means that transceivers are present at all interfaces to the switch, and these transceivers provide an OE (input) and EO (output) conversion of the signal. The presence of the transceivers at the edges of the switch fabric enables the switch to access the SONET/SDH overhead bytes for control and management functions. The opaque transceivers provide support for fault detection and isolation, performance monitoring, connection verification, neighbor/topology discovery and signaling, as well as support for implementing the network routing and restoration protocols.

The opaque switch approach was however faced with a number of challenges when confronted with the (unrealistic) traffic growth projections from just a few years ago: It would eventually reach scaling limitations in signal bit rate, switch matrix port count, and NE cost. These were the key motivations behind the attempt to develop large port-count transparent switches. For high-port count fabrics, analog gimbal-mirror (3D switches) MEMS-based switches offered the most viable approach $[12,13]$.

It is important to point out that the opaque switches may still remain in the network architecture in order to provide some key network functions, namely grooming and multiplexing, SLA verification, and control and management. If grooming and multiplexing functions are not required, it is possible to provide SLA verification, and control and management functions via a transparent switch with $\mathrm{O} / \mathrm{E}$ interfaces for the drop ports. 


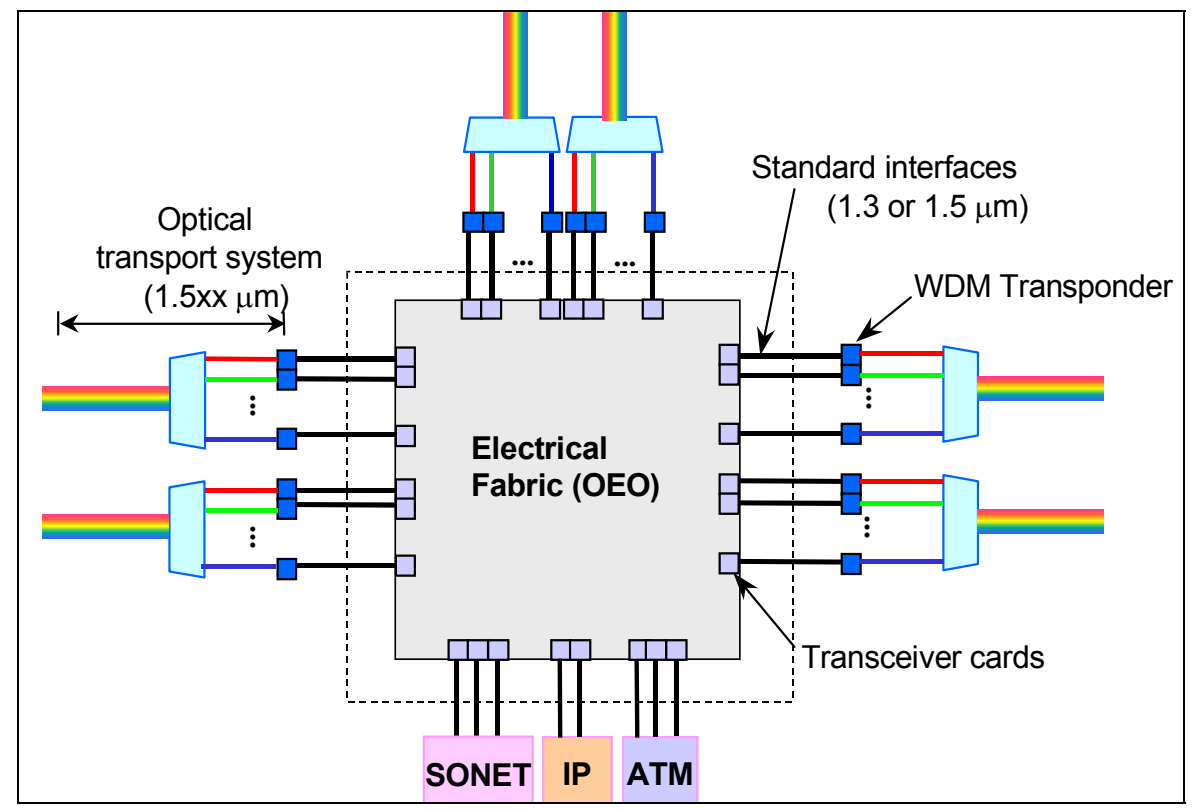

Figure 4: Opaque switch architecture

The promise of optical switching was that, unlike integrated electronic switches, an optical switch fabric's complexity is a flat function, independent of the bit rate of the signals it handles (Figure 5). Moreover, in the long run, it was projected that few components would be as small, cheap, and low in power-consumption as a silicon micro-mirror in the case of MEMS-based switch fabrics. Transparent switches could thus be expected to be cheaper in terms of the switching fabric and interface card cost compared to opaque switches ${ }^{3}$. This would then result in significant cost reduction to network operators because a large amount of the traffic that passes through an office will be able to bypass the OEO switch (typically approximately $75 \%$ through-to-total ratio). Transparent switches essentially help relieve the demand for OEO switch ports and reduce the cost of transporting lightpaths. This is accomplished by having all lightpaths pass-through (glass-through) the OOO switches, thus bypassing the OEO switches. Note that this can be a significant portion of the network traffic. For example, when OC-768 data rates are used, every time a lightpath passes through an OOO switch, 32 equivalent OC-48 ports of an OEO switch are saved (2 OC-768 ports that correspond to 32 equivalent OC-48 ports).

Since the switch fabric is bit-rate and data format independent, the switch matrix can scale more easily than electrical switch fabrics. For these reasons, as bit rates rise, optical switch

\footnotetext{
${ }^{3}$ However, this will not happen until a certain level of mass production of the switch fabric is achieved.
} 
fabrics may eventually prevail. But this would only have happened on time scales that are gated by the ability of vendors to meet carrier reliability and operational requirements with all-optical technologies such as lightwave micro-machine (for MEMS-based switch fabric) technology [12]. Even though in early stages of OC-48 and OC-192 development the crossover point shown in Figure 5 appeared to be at the OC-48 and then the OC-192 rates, under today's more realistic traffic growth scenario, and given the lack of deployment of 40 $\mathrm{Gb} / \mathrm{s}$ WDM systems and the continued decline in price of OEO components, the crossover point is shifted to the even higher bit rates. Furthermore, the need for and the promise of transparent switches appears to have moved beyond the foreseeable future.

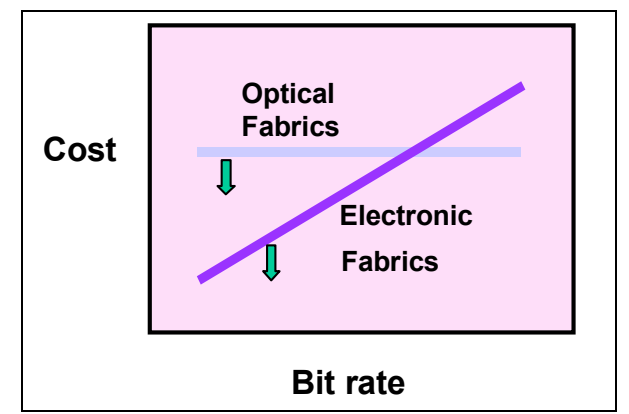

Figure 5: Advantages of Optical Fabrics

Figure 6 shows a transparent switch architecture. In this architecture, optical signals pass through e.g. a MEMS-based switch fabric in contrast to the OEO architectures where switching is accomplished using an electrical switch fabric. This switch architecture has transparent interface cards, i.e. no (OEO) transceiver (TR) cards are located at the network ports of the switch fabric that convert the optical into electrical signal. The switch shown in also has no opaque transceiver (TR) cards on its add/drop ports. Therefore, it has no direct access to the overhead bytes for control and signaling. The optical switch fabric is bit-rate independent and it accommodates any data rates available (e.g., OC-48, OC-192, OC-768). The advantage of such a switch architecture is that for an $\mathrm{NxN}$ OOO architecture there are $\mathrm{N}$ interfaces/ports to the OOO switch regardless of the type of interfaces. No data rate specific interface cards are used, so no replacement is needed when the switch is operating at higher data rates, provided that the optical power budget is sufficient for that rate. This is in contrast to the OEO systems where the number of ports depends on the type of the port. For example, in an OEO system one OC-192 interface card will replace four OC-48 interface cards. From 
the interface card perspective all ports in the OOO will look one and the same (same port cards are used for different signal rates and formats).

The drop-side ports are connected to an OEO switch (or any other client - such as IP or ATM) that provides SONET/SDH termination through its opaque ports. Note that integrating the opaque interfaces at the drop-side interfaces of the transparent switch can also provide the opaque function. $\mathrm{O} / \mathrm{E}$ drop interfaces in an $\mathrm{OOO}$ switch can be a cost-effective solution but cannot perform grooming or multiplexing functions. Thus, network level cost reduction may be achieved with two switches (an OOO and an OEO circuit switch and/or packet switch) even though the cost of two switches may be higher than the cost of an OOO switch with opaque drop-side interfaces. The decision to deploy two switches or one switch with opaque drop side interfaces is driven by the network needs and equipment availability. If the twoswitch approach is adopted, a communication interface between the OOO and OEO switches (e.g., an Ethernet external communication channel) may be required to perform control and management functions between the two switches.

The main challenge to architectures that use transparent switches is providing the control and management functionalities that are readily available when we have access to the electrical signal and consequently to the SONET/SDH overhead bytes. The section that follows focuses on the network control and management functions for an opaque network with opaque or transparent switches. 


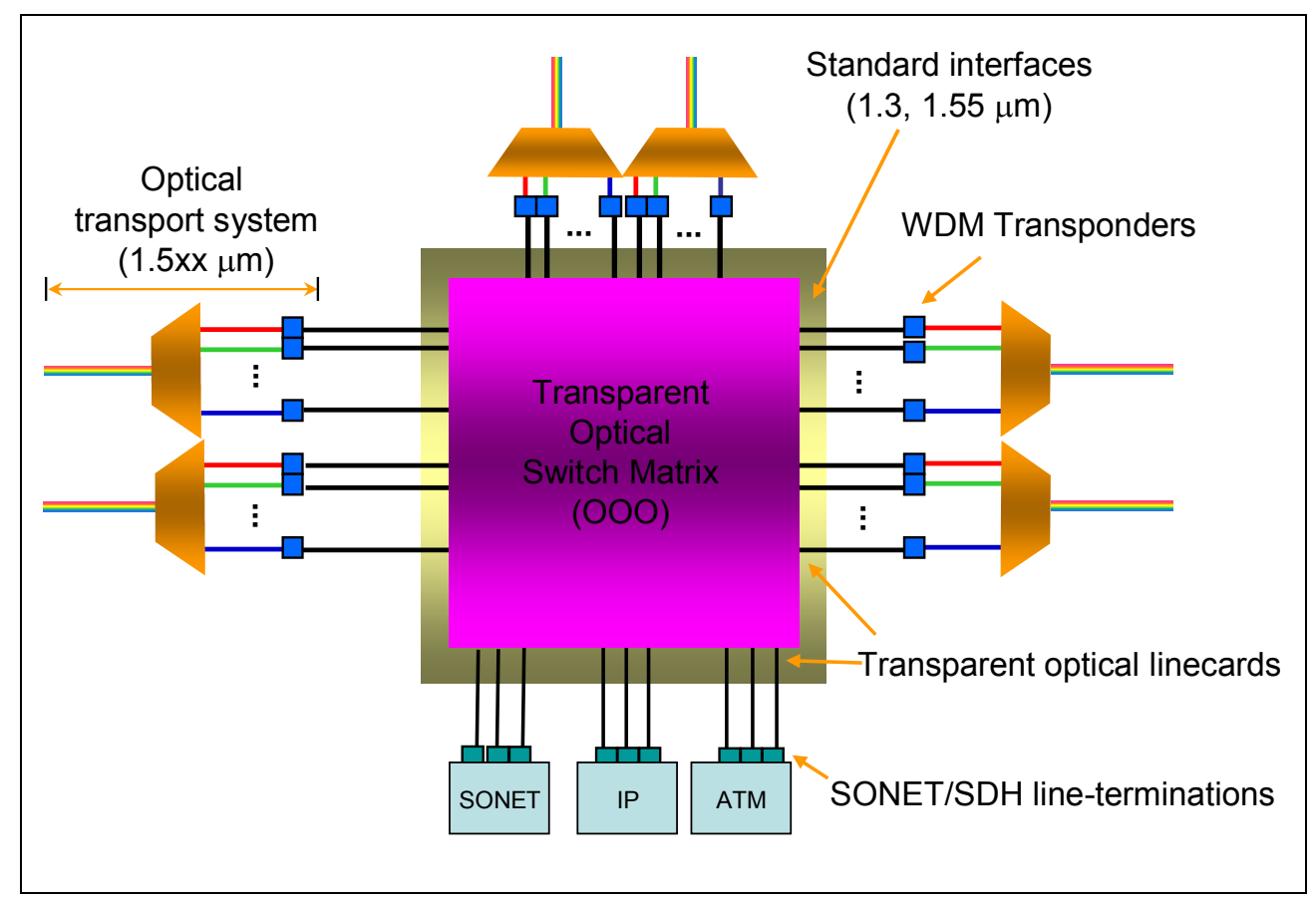

Figure 6: Transparent switch architecture

\section{Network Control and Management for Opaque Networks}

In order to build a dynamic, scalable, and manageable backbone network it is essential that manual configuration be eliminated as much as possible. This requires automatic neighbor and network topology discovery and other networking functions such as service assurance (e.g. access point performance monitoring for Service Level Agreement (SLA) verification), interworking with other network elements (e.g., keep-alive signal), fault management, and performance management regardless of the switching technology.

\subsection{With Opaque Switches}

Access to the SONET/SDH overhead bytes at the opaque interface cards is a key enabler of network control and management functionalities outlined below:

(1) It allows an opaque switch to perform in-band signaling and provisioning functions. Access to the overhead bytes also allows the switches to run dedicated mesh $(1+1)$ protection and shared mesh restoration protocols [4].

(2) It allows an opaque switch to run automated neighbor and topology discovery protocols. The ability of the network to autonomously create and maintain its resource databases is the fundamental building block for an efficient, flexible and manageable network. The 
neighbor discovery protocol using overhead bytes allows the network to create and maintain the critical port-state and topology databases. An automated neighbor discovery protocol is required in order to perform (a) node-port assignment discovery between the client and OEO switch architectures and (b) inter-office node-port associations between two neighboring OEO architectures. The OEO topologies are then created automatically by e.g. a management system that utilizes the node-port assignments between the client and OEO architectures and the inter-office node-port associations between neighboring OEO architectures found during the neighbor discovery process.

(3) All the network management functionalities also utilize the SONET/SDH overhead bytes at the interface cards. For example, fault detection and performance monitoring take place in the SONET/SDH processors located in the interface cards. Lightpath-based restoration or switch fabric protection switching can be triggered in the case of a detected failure condition. Fault isolation in such an architecture relies on the alarms generated by the switch interface cards after a failure is detected.

(4) In addition, read/write access to the SONET/SDH overhead enables the switch to provide service assurances such as performing connection verification and control to avoid misconnections.

(5) Finally, since the signal is terminated at each interface to the switch, an unequipped signal (keep-alive) is generated at every idle transceiver on the switch's line-side per SONET/SDH standards to prevent alarms in other equipment (WDM, router, etc) connected to the switch. This feature is required in switch architectures that implement shared mesh restoration since the channels have been provisioned but are not carrying any signal until a failure event occurs. Note that this feature is not needed on $1+1$ primary or backup lightpaths carrying live signal. In OEO systems the opaque interface cards generate an unequipped signal on the back-up channels of mesh-protected lightpaths. In the absence of a keep-alive signal, for example, all WDM transponders downstream could interpret the absence of signal as a signal-fail event, shut down the transponder lasers and generate a number of alarms. By always having a keep-alive signal through the OEO switch, equipment such as the WDM systems are prevented from generating any alarms. They are also prevented from having to fire-up lasers and perform power adjustments and equalization (which may require some manual intervention as well) 
when the channel is utilized (such as for a shared mesh restoration event). Thus, the time from when a failure is detected until the signal restoration is completed can be minimized.

\subsection{With Transparent Switches}

The lack of laser transmitters in a transparent switch and the lack of access to the electrical signal and consequently to the overhead bytes at the transparent switch interfaces pose a number of challenges in creating a seamless interoperable and manageable network. Network control and management features such as fault monitoring and localization, neighbor and topology discovery, SONET keep-alive generation, sophisticated signaling, performance monitoring and connection verification are collectively very difficult to achieve in a transparent switch without forfeiting the economies that the switch was designed to extract. Approaches to address these challenges principally consist in either deploying OEO cards on the drop side of a transparent switch, or in using and/or relying on the OEO function located at WDM transponders and/or at the client equipment OEO switches, effectively using these equipment as proxies. Several examples of support for network management and control functions are discussed below:

\section{(a) Automatic Port/Neighbor and Topology Discovery}

Automatic port/neighbor discovery and topology discovery are key aspects of a service provider requirements. The Link Management Protocol (LMP) standard [14] has been proposed to automatically discover (a) node-port associations between the OEO client and the OOO switches and (b) inter-office node-port associations between two neighboring OOO switches. LMP handles transparent switches by using dedicated opaque cards temporarily or the opaque interfaces on the OEO clients and out-of-band signaling to discover connectivity between switches. After the LMP protocol is run and the node-port associations are ascertained, the network topology can be created automatically by a centralized management system or in a distributed way.

If LMP cannot be implemented, a proprietary procedure can be designed for port/neighbor discovery. Such a procedure could be manual or automated. The manual procedure would entail initial discovery of the neighbor and port adjacency relationships and implicit continuous checking of these relationships since the channels will be part of provisioned 
lightpaths or of an unequipped signal loop-back chain as described in the next section. In both cases, a change in the adjacency relationship will result in failure of the lightpath or the loop-back chain, thereby detecting the problem. Additional procedures will then have be used to localize the incorrect adjacency relationship. In order to automate this process, the OOO switch has to be equipped with a small number of opaque interface cards providing OC-n signal generation functions. These cards will be used to conduct neighbor/port discovery in an automated process by establishing a connection between the opaque interface cards of two adjacent OOO switches as shown in Figure 7.

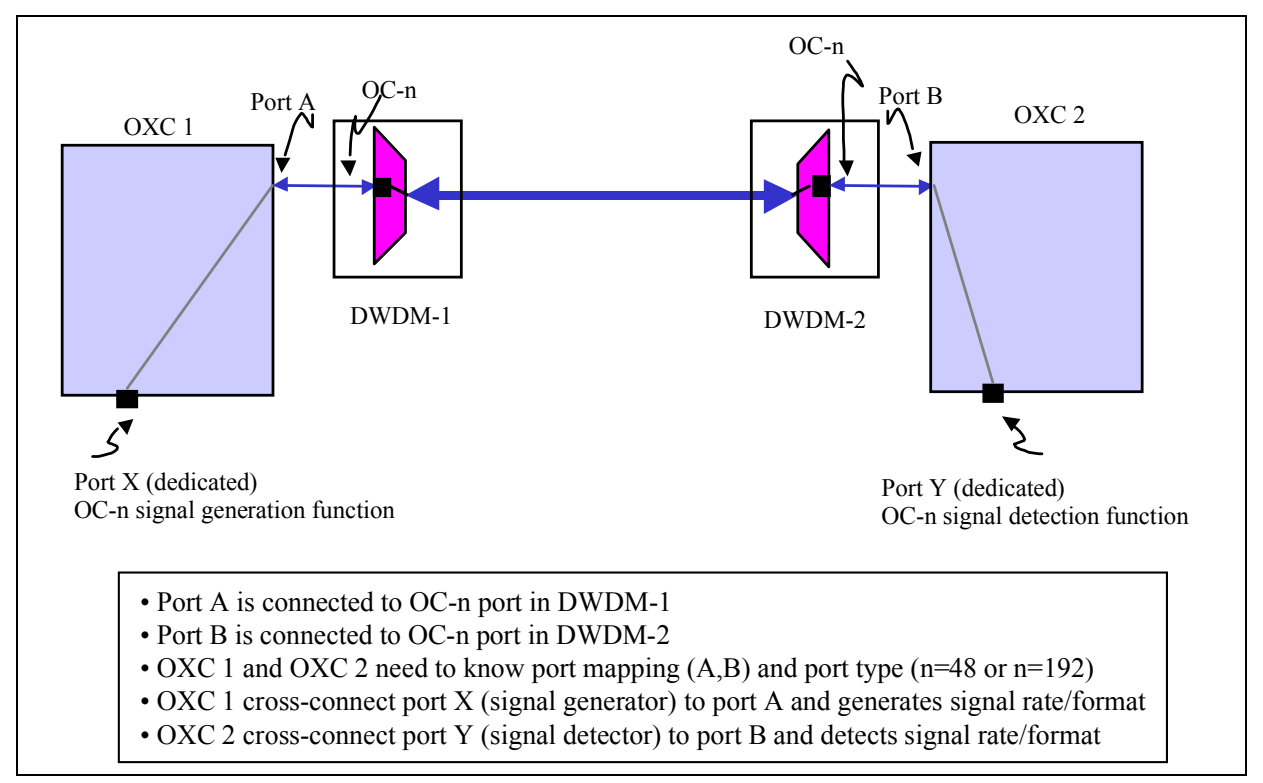

Figure 7: Use of opaque cards for automated neighbor and port discovery

\section{An Automated Neighbor Discovery Process}

Upon connecting a transparent interface port on the OOO switch to a WDM transponder, the port status in the switch is changed (via a craftsperson) to a new state called "ready-todiscover". Upon such change of port status or at pre-defined times, the automated neighbor/port discovery process will be implemented. There are two approaches to carrying out the process. In a centralized approach, the process will be driven by an Element Management System (EMS) instructing the switches to carry out the required operations. In a distributed approach the switches would carry out the required operations on their own, communicating with each other over the switch communication infrastructure. More details on such approaches are presented below: 
In the centralized approach, the EMS would instruct a switch, e.g. $\mathrm{OXC}_{1}$, to connect the OC$\mathrm{n}$ signal generation port to one of the "ready-to-discover" ports. It then instructs another switch, say $\mathrm{OXC}_{2}$, to set up cross-connects between the OC-n signal detection port and the "ready-to-discover" ports, one after the other, until the signal sent by $\mathrm{OXC}_{1}$ is detected. If such a signal is detected, $\mathrm{OXC}_{2}$ reports this to EMS, which derives the neighbor/port adjacency relationship and informs the two nodes of it. If the appropriate signal is not detected on any of $\mathrm{OXC}_{2}$ 's "ready-to-discover" ports, EMS instructs another switch, say $\mathrm{OXC}_{3}$, to carry out a similar procedure. This procedure is repeated until a signal from $\mathrm{OXC}_{1}$ is detected, yielding a neighbor/port association or all the "ready-to-discover" ports at all switches have been tried. If no association is discovered, the port on $\mathrm{OXC}_{1}$ remains in the "ready-to-discover" state. Note that this procedure could be carried out in parallel at $\mathrm{OXC}_{2}$ and $\mathrm{OXC}_{3}$, and any other switch with "ready to discover" ports to speed up the discovery process.

In the distributed approach, $\mathrm{OXC}_{1}$ is communicating with all other $\mathrm{OXCs}$ to determine if they have ports in the "ready-to-discover" state. If they have, the corresponding OXC goes through the procedure of cross-connecting its signal detection port to every "ready-todiscover" port, each in turn. If the signal is correctly received, $\mathrm{OXC}_{2}$ communicates its identity and that of the port to $\mathrm{OXC}_{1}$. In turn, $\mathrm{OXC}_{1}$ communicates the corresponding information to $\mathrm{OXC}_{2}$. Both switches then create the appropriate entry into their neighbor/port adjacency database. Such updates are then communicated to EMS. Note that the entire procedure could be carried out at $\mathrm{OXC}_{2}$ and $\mathrm{OXC}_{3}$, and any other $\mathrm{OXC}$, in parallel, thus speeding the whole process. 


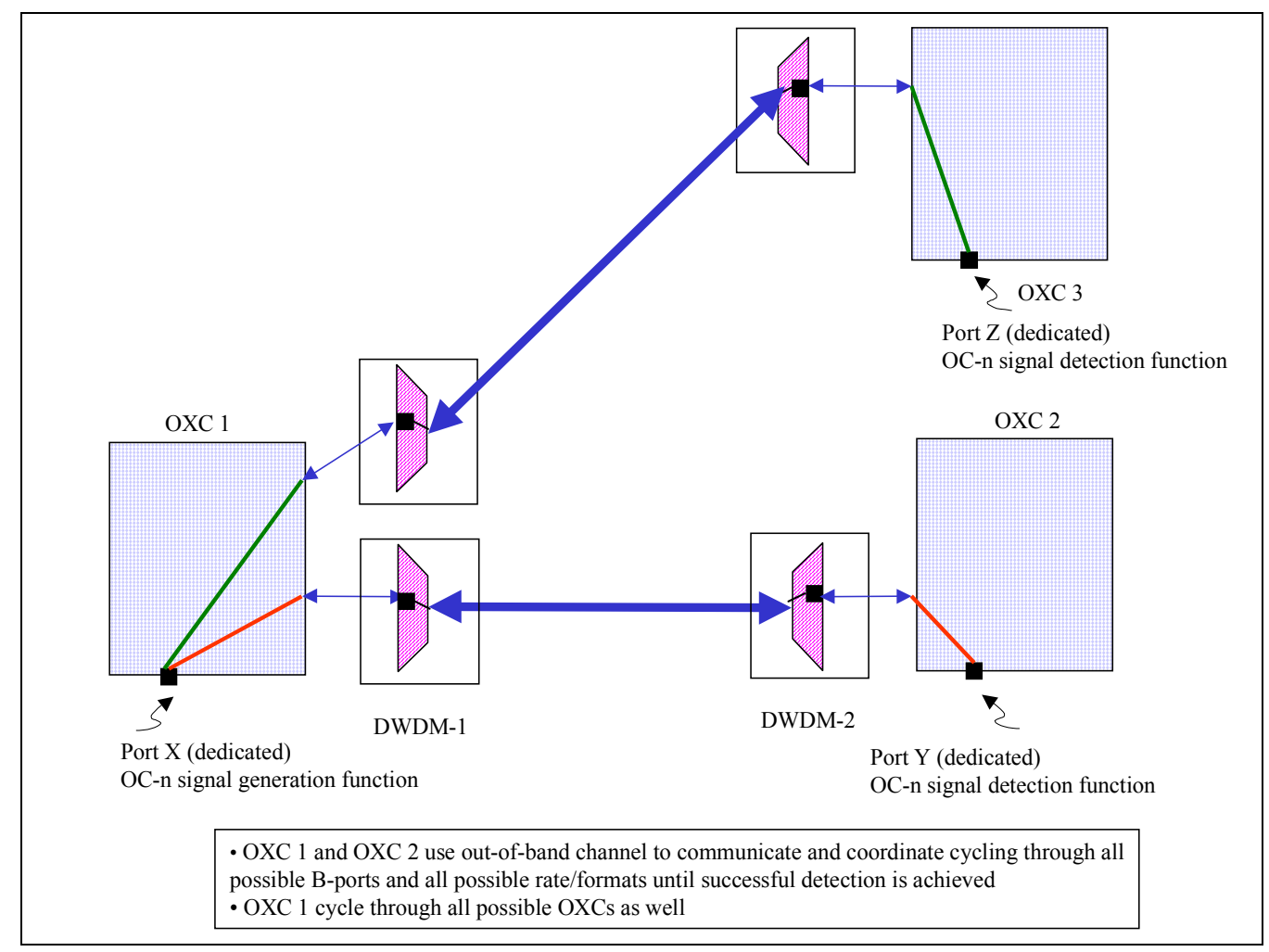

Figure 8: Cycling through all possible OXCs and ports

This procedure may also require trying the different opaque transceiver speeds using the same technique as described above (centralized or distributed). Since this process happens infrequently, there are no real-time requirements on creating those adjacency relationships. As in the case of OEO switches, topologies comprised of OOO switches are then created automatically by e.g. a management system that utilizes the node-port assignments between the client and $\mathrm{OOO}$ architectures and the inter-office node-port associations between neighboring $\mathrm{OOO}$ architectures found during the neighbor discovery process.

Because of the nature of all-optical switches, the proposed architecture and protocol for neighbor discovery will allow for an initial discovery of the neighbor and port adjacency relationship but will not continuously and specifically check that the adjacency relationship is maintained as is the case with the OEO switches. Instead, some continuous checking will be done implicitly since the channels will be part of provisioned lightpaths. A change in the adjacency relationship will result in failure of the lightpath, thereby detecting the problem. Procedures will then have be used to localize the incorrect adjacency relationship. 


\section{(b) Provisioning and Network Protection and Restoration}

A-to-Z provisioning and signaling is done in a centralized or distributed approach and can be triggered by an operator (point-and-click A to Z provisioning) or by UNI/GMPLS signaling through the OEO function [15-17].

Network protection and restoration can be provided in two different ways. Protection and restoration can be supported entirely through the OEO clients of the transparent switches. In this case, the transparent switches are not involved in the protection and restoration process. All the lightpaths and shared back-up channels effectively terminate on the OEO clients. Alternatively, protection and restoration can be supported entirely within the transparent switch-based network, with lightpaths and shared back-up channels terminating on transparent switches ${ }^{4}$. The restoration crossconnects are then performed at the transparent switches upon appropriate triggering (such as SD or SF conditions) by the OEO switches, for example, through a control link.

A network of transparent and opaque switches (see ) provides dedicated (1+1) as well as shared-mesh restoration capabilities [4]. ). Figure 9 below shows an example of a network of interconnected $\mathrm{OOO}$ architectures (via their network ports) that have client architectures connected to their add/drop ports. The channels on the backup path of the shared-mesh restored lightpaths terminate at an $\mathrm{OOO}$ switch (for additional port savings at both the $\mathrm{OOO}$ and OEO switches). In this example, the OOO switches do not depend on the clients to provide the restoration function. Rather, they takes active part in the restoration, by reconfiguring their switch fabrics in order to route the backup path.

\footnotetext{
${ }^{4}$ Some form of local protection can be supported between the OEO client and a transparent switch within an office, complementing the network protection/restoration scheme.
} 


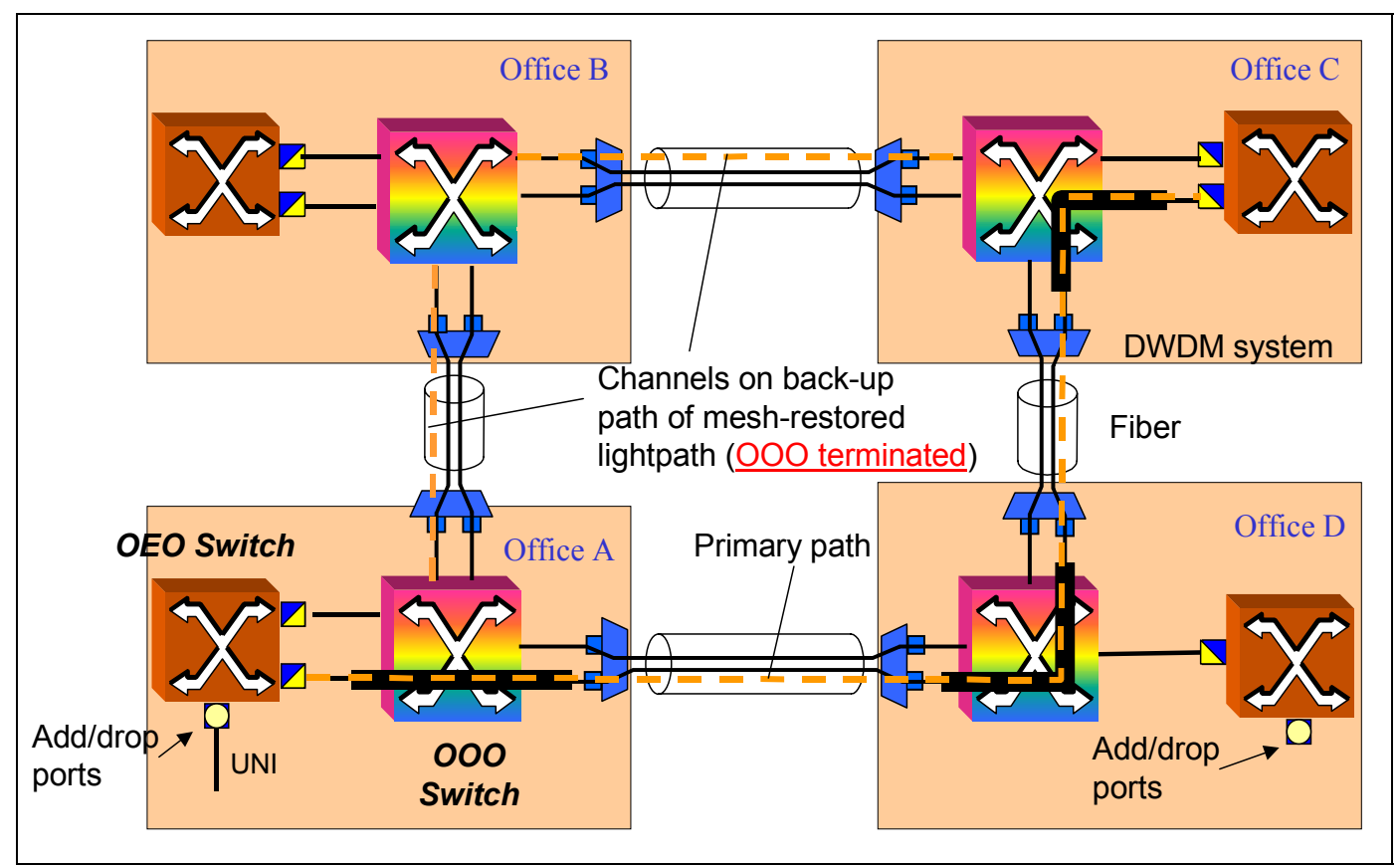

Figure 9: Mesh restoration example

Restoration triggering and restoration signaling in OOO-based restoration are the two schemes that will be different from the ones used in OEO-based restoration. An out-of-band signaling scheme will now be required to support OOO-based restoration (e.g., through a router-based DCN network). Special care must be taken to ensure that the DCN network used is reliable and allows for the transfer of the restoration messages in a reasonable amount of time.

A number of restoration triggers can also be used. For example, the failure can be detected at the WDM system and a communication protocols between the OXC and WDM systems can be used to trigger restoration at the OOO switch [18-20]. The failure can also be detected at the client's interface and an out-of-band control channel (possibly through an extension to UNI 1.0 [21], a new UNI 2.0, or a proprietary scheme) between the OOO switches and the client drop nodes can be used to trigger restoration at the OOO switches. An out-of-band signaling channel would also be required between the OOO end-nodes and the client drop nodes to support a protection protocol between the switch and the drop nodes (e.g., using UNI 2.0). Note that a "handshaking" protocol between the client drop node and the OOO end-node is required in order to accommodate the interplay between network restoration and the switch-client node protection protocol. 
The restoration approach described in this section is a lower cost approach compared to the restoration in opaque networks with opaque switches, as it saves a number of OEO ports. However, it offers slower shared mesh restoration with out-of-band signaling, and it requires new out-of-band OOO-to-OOO and OOO-to-client signaling channels. Note that OOO-toclient out-of-band signaling will also require vendor cooperation and new standards definition.

\section{(c) Performance Monitoring}

Optical performance monitoring (OPM) can take place at the transparent switch interfaces in the form of optical power monitoring. But electrical performance monitoring can only take place at the OEO endpoints of a lightpath (using the SONET/SDH processors in an opaque card), since there is no signal visibility on transparent switches (lightpath-based PM).

\section{(d) Connection Verification}

The transparent switch architecture provides end-to-end lightpath connection verification at the lightpath endpoints utilizing the lightpath ID. Mirror connection (mirror-port association), in the case of MEMS-based switch fabrics, and port-fiber association verification can also be used in order to localize misconnections.

\section{(e) Fault Detection and Fault Localization}

Fault detection (other than Loss-Of-Light (LOL) that is monitored at the transparent interfaces of the switch) takes place in the opaque interfaces located at the lightpath endpoints. A failure must be detected and isolated to the level of a replaceable unit. The fault isolation need not be an instantaneous process. As long as the failure is detected (at the lightpath end-points) and a restoration mechanism is triggered quickly, fault isolation can be a slower process. With transparent switches, fault localization can take place at the management system by correlating the alarm information generated by the switches. In some cases, fault localization may require alarms generated by the WDM systems as well. In that case, one could essentially use the transponder's access to the electrical signal as a proxy for opaque interfaces in support of control and management functions. In addition, the usage of sequential loopback can support fault localization, when it is not possible via alarm correlation, as proposed with LMP. Looking ahead, if a communication channel between the switches and the WDM systems is implemented, fault localization is expected to be a simpler 
process. The IETF and the ITU have been considering communication protocols between the OXC and WDM systems to support control and management functions [18-20].

\section{(g) Unequipped-Signal Generation, Distribution and Maintenance}

In this OOO-switch architecture, to be capable of providing shared mesh restoration, the provisioned mesh-restored channels (when not in use) require the presence of an unequipped (keep-alive) signal. This is true because the lack of an unequipped signal results in (a) alarms generated at the WDM systems that have knowledge of provisioned channels but detect no light on those channels, (b) lack of monitoring of the restoration channels to ensure availability when or if a failure occurs and (c) increased restoration time if a failure occurs, due to the additional time required to turn on the ITU grid WDM lasers. Unequipped signal generation becomes an issue in the case of transparent switches, since the (transparent) interface ports cannot inject an unequipped signal. Otherwise, a laser would be required at each line-side port, which defeats the purpose of the all-optical switch. A new method of providing an unequipped (keep-alive) signal is thus required. This can be achieved by "filling" all idle channels (unused and reserved for restoration) with a limited number of lasers as is explained below. In all the proposed techniques laser signals are injected from the drop sides of optical switches, and each signal propagates along several idle channels, looping back and forth between the switches.

The proposed scheme equips the OOO switches with additional opaque transceivers (TRs) so that all the provisioned ports (those not carrying lightpaths and those used for restoration channels) between two OOO switches can be looped back through the fabric, thereby creating an unequipped signal on the ports. This approach requires as many opaque TRs, as roughly half the average node degree per OOO plus possibly a few additional cards for redundancy purposes. The cards can be added as needed at an OOO switch as the degree of that node increases. Note that this approach also mitigates the risk of silent failure in a simplex fabric, or on the working fabric path for a shared mesh channel in a duplex fabric.

There are two ways, shown in Figure 10, to inject unequipped signals into a network. One is to use the TR of the OEO functionality at the add/drop ports of each node to generate and to monitor the keep-alive-signal. The advantages of this approach are that there is no need for separate laser sources, monitors and the software to manage them. Another approach is to use 
a separate laser source and monitor, and to connect them directly to the OOO switch. This approach saves one client port, but loses the corresponding advantages as well.

The SONET header of a keep-alive-signal contains the address of the originating node followed by the address of the looped-back node as the ID of the keep-alive-signal.

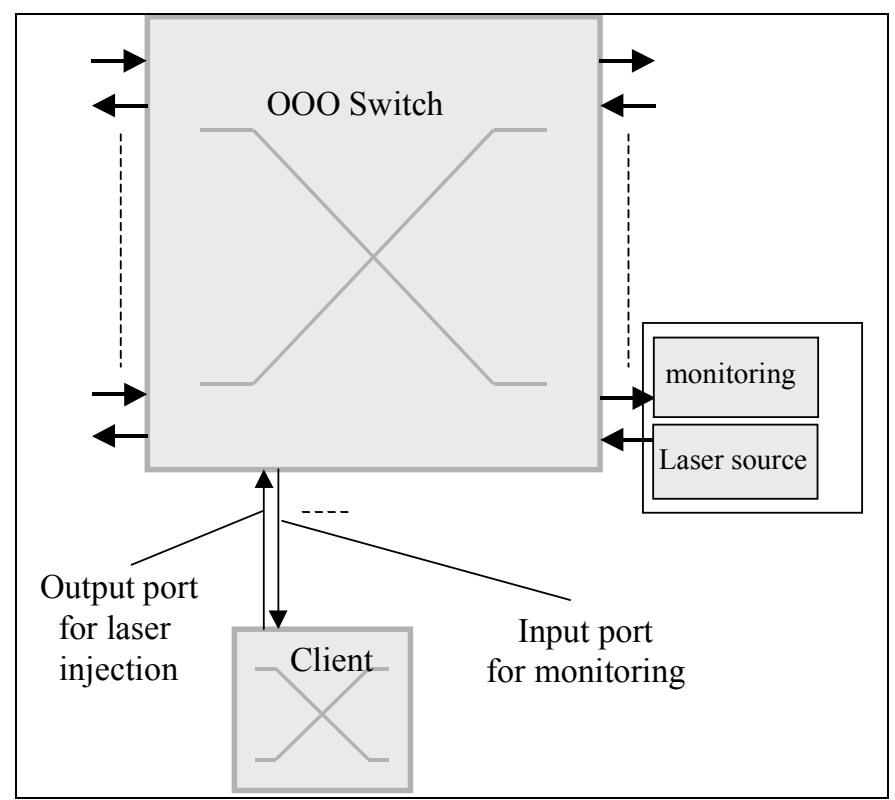

\section{Figure 10: Unequipped signal injection and monitoring}

\section{Unequipped-Signal Generation}

Keep-alive-signals are sent to all the shared mesh restoration channels not in use, and to all the working channels not in use. Consider a keep-alive-network that consists of all those notin-use channels as in Figure 11. The two node addresses forming the keep-alive-signal ID can be used to establish an order in the network. In the case where all keep-alive laser sources are working, the keep-alive-signal can be sent from the node with the smaller ID to the node with the larger ID in a looped-back chain as shown in Figure 11, and the signal travels only one span from the source node. A keep-alive-signal always comes back to the same node from which it is sent. The numbers marked along the spans in Figure 11 show the source of each keep-alive-signal. Each node knows all the channels that are not used, and are attached to it. Among those channels, it also knows to which channels it should send its own keep-alive-signal, and on which channel it should expect the keep-alive-signal to be sent by a neighboring node. Finally, the nodes on a switch facing a given switch need to be crossconnected appropriately as part of the loop-back chain to avoid cycles which would not carry 
the unequipped signal. This can be accomplished in several ways. One scheme would have all loop-back cross-connects at a switch made in increasing order of port ID. Another scheme would trigger the cross-connect on detecting light coming in (OPM functionality), then completing the loop-back cross-connect according to the increasing port ID scheme. This technique allows the switches to autonomously set the loop-back cross-connects, since after each cross-connect, one of the switches will see light on one its provisioned port. A problem with this technique arises if some WDM systems insert an unequipped signal even if they are not receiving a signal from the OOO switch.

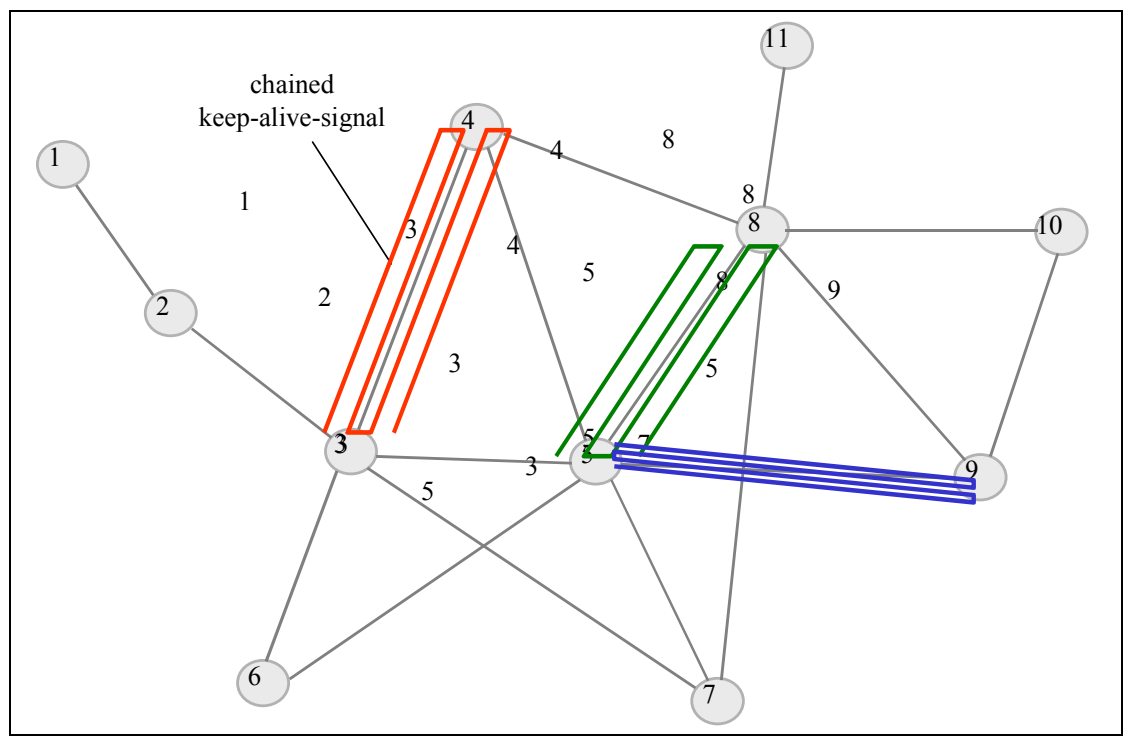

Figure 11: Unequipped signal distribution

\section{Unequipped signal maintenance}

The two node loop-back chains would change in the following situations:

- A new port pair is added

- A port pair is used for provisioning service

- A port pair is used for restoration of a shared mesh restorable service

- A port pair is used for re-provisioning 


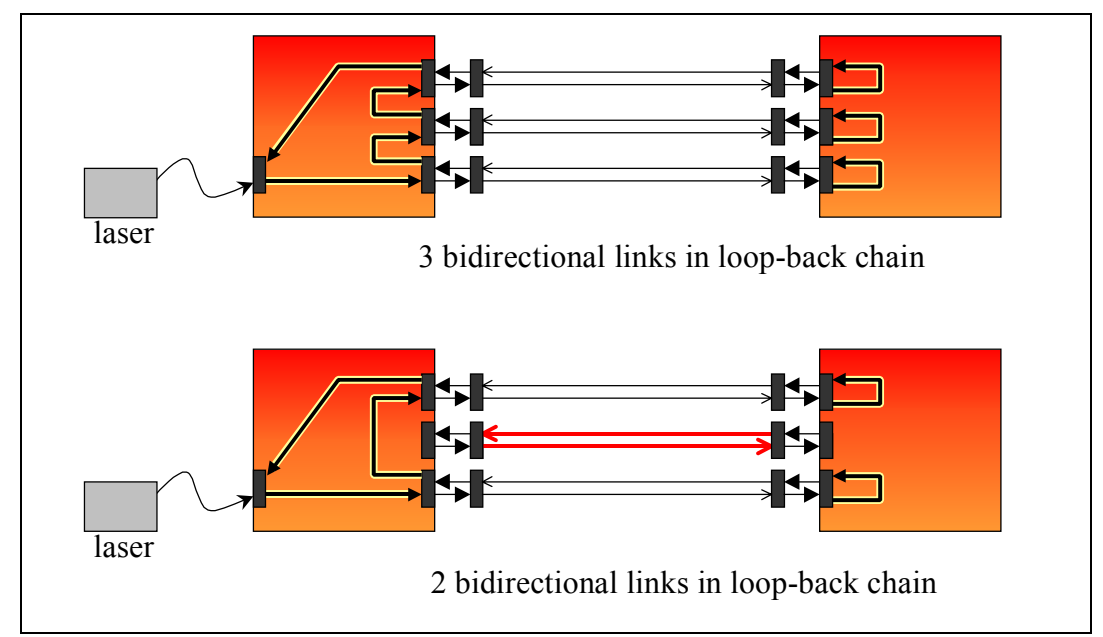

Figure 12: Maintenance of unequipped signal loop-back chain

A complication in this scheme arises when a channel in the loopback chain is seized for shared mesh restoration. When this happens, the loopback chain could be open for a few tens of msecs (time it takes to reconfigure the switch fabric and create a new loopback chain). During that time, alarms may be raised from the WDM equipment and the WDM lasers can shut down. Alarms are not considered a major problem, as most WDM equipment have a soak time that is more than $10 \mathrm{msec}$ before they issue an alarm. Even if an alarm is raised, it can be suppressed by the EMS/NMS. The problem of the lasers shutting down is a more serious one as it can have a direct impact on the restoration times for the backup channel, as well as an impact on the other channels on that path (if channel power equalization is required once the lasers are turned back on). We are currently investigating how the WDM systems behave for provisioned channels with no optical signal.

Note that if a dedicated opaque card is not developed, an out-of-band communication scheme between the OXCs and the WDM systems could be used to "work around" the keep-alive signal generation issues by suppressing alarms and keeping the WDM lasers on even in the absence of keep-alive signals for provisioned but not in use channels. Standardization work is currently under way in the standardization bodies to develop a communication protocol between the OXC and WDM systems that could successfully be used to address this problem [18-20]. 


\section{(h) Power Budget Management}

An important operational issue associated with a transparent switch is power budget management. Because of the relatively high insertion loss of the optical switch fabrics and the resulting loss from input to output port, traditionally deployed cross-office optics cannot be supported with a transparent switch. Therefore, such architectures require higher-cost cross-office optics or new low-cost ones currently being worked on in OIF [22].

\begin{tabular}{|l|c|c|c|c|c|c|}
\hline & VSR & SR-1 & SR-2 & IR-1 & Hi-P VSR & LR-1 \\
\hline $\begin{array}{l}\text { Standards } \\
\text { Body }\end{array}$ & OIF & Bellcore & Bellcore & Bellcore & OIF & Bellcore \\
\hline Length (km) & 0.6 & 12 & 20 & 24 & 0.6 & 48 \\
\hline $\begin{array}{l}\text { Nominal } \\
\text { Wavelength (nm) }\end{array}$ & $1290-1330$ & $1290-1330$ & $1530-1565$ & $1290-1330$ & $1290-1330$ & $1290-1330$ \\
\hline Attenuation (dB) & $4+1$ & $6+1$ & $6+2$ & $11+1$ & $11+1$ & 22 \\
\hline $\begin{array}{l}\text { Optical Path } \\
\text { Penalty (dB) }\end{array}$ & 1 & 1 & 2 & 1 & 1 & \\
\hline $\begin{array}{l}\text { Unit Power } \\
\text { Consumption (W) }\end{array}$ & 6 & \multicolumn{2}{|c|}{7.5} & 11 & 6 & 13 \\
\hline
\end{tabular}

Table 1: Cross-office Standard Interfaces (10 Gb/s)

\section{Switch Fabric for Transparent Switches}

Let us now consider the technology that will provide switch fabrics for all-optical switches. Micro-electromechanical systems (MEMS) offer the most promising means of building the high-port-count switch fabrics that are needed for the core-network cross-connects $[12,13]$. MEMS flip-up mirror arrays (2D switches) offer the potential for small $(<32)$ port count OXCs [23-25]. Analog gimbal-mirror (3D switches) MEMS-based switches offer the most viable approach to make a single-stage switch with port counts numbering from the hundreds to thousands.

Figure 13 shows how a beam of light is switched from an input to an output port using such a fabric. In these fabrics, two matrices of gimbal-mounted MEMS mirrors with 2-axis control are used to provide beam switching in a $3 \mathrm{D}$ volume between two $2 \mathrm{D}$ arrays of collimated fibers. The first demonstration of a MEMS device and system prototype for this type of OXC was the Texas Instruments/Astarte Fiber Networks' Beehive switch [26], though the device technology achieved in this program was rapidly improved upon by many others during the 
last few years. The ability of this architecture to achieve large port counts, while retaining reasonable optical performance, is the primary driver for these systems.

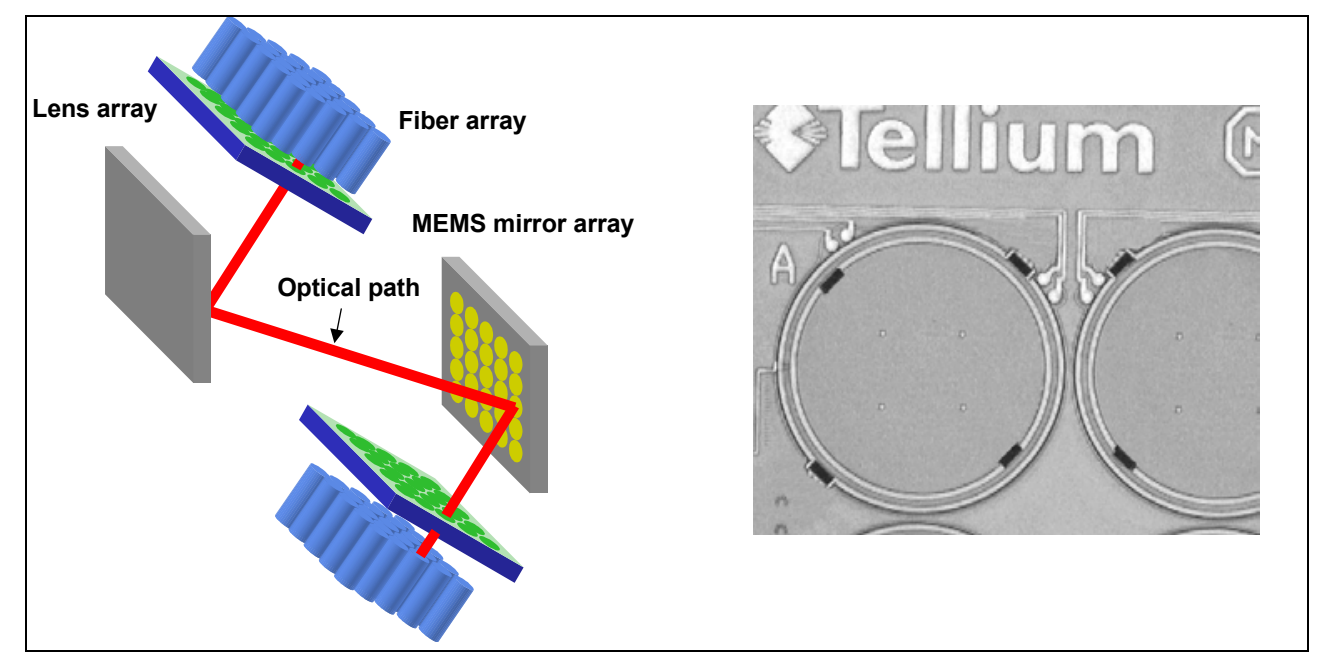

Figure 13: a) Schematic of a 3D MEMS OXC architecture. b) Photograph of a gimbal-mounted 2-axis MEMS mirror.

Although the public perception of MEMS switches is that they are real and ready for shipping, the reality is actually very different. Reliability qualification of MEMS switches is still ongoing, though some levels of NEBS and other forms of certification are being achieved. At present, no service-grade field-deployable large port-count fabrics have been shipped by any company, and efforts in this area have slowed due to diminished demand. The fundamental challenges faced in these systems, specifically in the areas of MEMS chips, optical fiber arrays, lenslet arrays, mirror-position control systems and packaging subsystems is discussed in [27]. The specifics and complexity of each of these components depends dramatically upon the size and configuration of the cross-connect itself, and presents significant challenges to the succesful deployment of these network elements.

\section{Role of Transparency in the Near Future}

As previously mentioned, the applications and the traffic demand driving the large scale deployment of transparent optical switches in the core network are not currently in place. However, even though the use of transparent switches in the core that are cost effective at very high bit rates is not currently justified, there still exist some niche applications in today's networks that use a small number of transparent switches. 
Based on the challenges outlined in the previous sections and the forecast traffic demand and near term applications that will be supported, it is anticipated that in the near future transparency will be first limited in metropolitan area networks, utilizing Reconfigurable Optical Add/Drop Multiplexers (ROADMs), and some ultra long-haul applications in the core, utilizing a small number of wavelength-selective cross-connects/OADMs on high capacity routes. When OADMs are utilized, selected wavelengths can be added or dropped at a node while the rest of the wavelengths pass through without regeneration $[28,29]$. Reconfigurable Optical Add Drop Multiplexers (ROADMs) further enable any user to access any channel. ROADMs can be utilized in metropolitan area networks at central offices and customer locations in much the same way as the SONET introduction created a need for large numbers of SONET ADMs. They provide network flexibility and can be used to manage continuous changing traffic patterns and customer service requirements.

Wavelength-selective cross-connects can also be used in ultra long-haul applications in the core network in a completely transparent manner. Even though these network elements allow for end-to-end bit rate and data format transparency, as previously outlined, they face a number of challenges. However, in the near future it is expected that these network elements will be utilized in a few, predetermined and non-reconfigurable high capacity routes to provide end-to-end transparency between fixed end-nodes.

\section{Conclusion}

The current state of affairs in terms of network deployment, applications and traffic demand does not justify the large scale use of transparent switches in today's networks. Some niche applications do exist, but can mostly be addressed using a number of small transparent switches. Provided that the traffic grows and the bit rates increase substantially there may emerge a potential need for an additional network layer utilizing transparent optical switches. Even from the technology standpoint, the state of 3D MEMS-based switches was at best "advanced development" for 256 port fabrics and "advanced research" for 1,000 port fabrics, even before most of the efforts in this area were halted due to diminished demand. In the meantime, the deployment of transparent network elements is expected to remain limited to wavelength selective cross-connect (WSXC) architectures and Reconfigurable Optical Add Drop Multiplexers (ROADMs) on high capacity routes. 
Furthermore, we anticipate that opaque switches will always remain for the embedded service base even after the transparent switches are eventually introduced in the network. These opaque switches will provide the grooming and multiplexing functions, as well as some of the necessary control and management functions, and will scale and decrease in cost with rapid progress in electronics.

While completely transparent core mesh networks are still far off on the horizon, even transparent switches in opaque networks still face technological as well as control and management challenges. Even though most of these issues can be addressed via clever innovation as well as standardization efforts, transparent switches complemented by an opaque function will not be ready for deployment in the network until all the challenges addressed in this paper are succesfully resolved.

\section{References}

1. T. Stern, K. Bala, Multiwavelength Optical Networks: A Layered Approach, Prentice Hall, May 1999.

2. K. Bala, et al, "The case for opaque multiwavelength lightwave networks", IEEE/LEOS Summer Topical Meeting, 1995.

3. B. Mukherjee, "WDM Optical Communication Networks: Progress and Challenges", IEEE Journal on Selected Areas in Communications, vol. 18, no. 10, pp. 1810-1824, October 2000.

4. G. Ellinas, et al., "Routing and Restoration Architectures in Mesh Optical Networks", Optical Networks Magazine, Issue 4:1, January/February 2003.

5. J. Labourdette, et al., "Routing Strategies for Capacity-Efficient and Fast-Restorable Mesh Optical Networks", Photonic Network Communications, vol. 4, no. 3-4, pp. 219-235, 2002.

6. E. Bouillet, et al., "Addressing Transparency in DWDM Mesh Survivable Networks", in Proc. OFC '01, Anaheim, CA, March 2001.

7. B. Ramamurthy, et al, "Impact of transmission impairments on the teletraffic performance of wavelength-routed optical networks," IEEE/OSA J. Lightwave Technol., vol. 17, pp. 17131723, Oct. 1999.

8. C. S. Li et al., "Gain equalization in metropolitan and wide area optical networks using optical amplifiers," in Proc. IEEE Infocom'94, Toronto, Canada, July 1994, pp. 130-137.

9. B. Ramamurthy, et al, "Optimizing amplifier placements in a multi-wavelength optical LAN/MAN: The equally-powered-wavelengths case," IEEE/OSA J. Lightwave Technol., vol. 16, pp. 1560-1569, Sept. 1998.

10. B. Ramamurthy, et al, "Transparent vs. opaque vs. translucent wavelength-routed optical networks," in Proc. Optical Fiber Communications. (OFC'99), San Diego, CA, Feb. 1999, Paper TuF2, pp. 59-61.

11. J. Strand, A. Chiu, and R. Tkach, "Issues for Routing in the Optical Layer", IEEE Communications Magazine, pp. 81-87, February 2001.

12. J. Walker, "The Future of MEMS in Telecommunication Networks", J. Micromech. Microeng., vol. 10, pp. R1-R7, 2000. 
13. J. E. Ford and J. A. Walker, "Dynamic spectral power equalization using micro-mechanics," IEEE Photonics Technol. Lett., vol. 10, pp. 1440-1442, 1998.

14. "Link Management Protocol (LMP)", IETF Draft, draft-ietf-ccamp-lmp-09.txt, June 2003.

15. "User Network Interface (UNI) 1.0 Signaling Specification", OIF contribution OIF2000.125.6, September 2001.

16. "Generalized MPLS - Signaling Functional Description", IETF Draft, draft-ietf-mplsgeneralized-signaling-05.txt, January 2001.

17. "Generalized MPLS Signaling - RSVP-TE Extensions", IETF Draft, draft-ietf-mplsgeneralized-rsvp-te-04.txt, January 2001.

18. "Link Management Protocol (LMP) for Dense Wavelength Division Multiplexing (DWDM) Optical Line Systems", IETF Draft, draft-ietf-ccamp-lmp-wdm-02.txt, March 2003.

19. "Optical Link Interface Requirements", IETF Draft, draft-ietf-ccamp-oli-reqts-00.txt, February 2002.

20. "Physical interface and topology aspects of VBI", ITU-T Contribution, October 2001.

21. "OIF User Network Interface Protocol”, OIF Contribution, oif2000.125.4, April 2001.

22. J. Ojha, "OIF Very Short Reach (VSR) Interface", OIF White Paper, www.oiforum.com.

23. L. Y. Lin, E. L. Goldstein, and R. W. Tkach, "Free-space micromachined optical switches with submillisecond switching time for large-scale optical crossconnects," IEEE Photonics Technol. Lett., vol. 10, pp. 525-527, 1998.

24. R. T. Chen, H. Nguyen, and M. C. Wu, "A low voltage micromachined optical switch by stress-induced bending," 12th IEEE International Conference on Micro Electro Mechanical Systems, Orlando, FL, 1999.

25. B. Behin, K. Y. Lau, and R. S. Muller, "Magnetically Actuated micromirrors for fiber-optic switching," Solid-State Sensor and Actuator Workshop, Hilton Head Island, SC, 1998.

26. H. Laor, "MEMS mirrors application in optical cross-connects," IEEE LEOS Summer Topical Meetings, Monterey, CA, 1998.

27. G. Ellinas, J-F. Labourdette, S. Chaudhuri, L. Lin, E. Goldstein, J. Walker, and K. Bala, "Transparent Optical Switches: Technology Issues and Challenges", IEC 2003 Annual Communications Review, 2003.

28. J. Bayne and M. Sharma, "Broadcast-and-select OADM enables low-cost transparency", Lightwave Magazine, December 2001.

29. J-K. Rhee, I. Tomkos, "A Broadcast-and-select OADM optical network with dedicated optical-channel protection", IEEE Journal of Lightwave Technology, vol. 21, no. 1, January 2003. 